\title{
RELAÇÃO DAS PROPRIEDADES MECÂNICAS E DENSIDADE DE POSTES DE MADEIRA DE EUCALIPTO COM SEU ESTADO DE DETERIORAÇÃO ${ }^{1}$
}

\author{
Rafael Colombo Abruzzi ${ }^{2}$, Marçal Rodrigues Pires ${ }^{3}$, Berenice Anina Dedavid ${ }^{4}$ e Silvia Baptista Kalil ${ }^{5}$
}

\begin{abstract}
RESUMO - Este trabalho apresenta correlações obtidas entre propriedades mecânicas, densidade e o estado de deterioração de postes de eucalipto novos e retirados de serviço. O estado de deterioração foi determinado por inspeções em campo de postes da rede de distribuição de energia elétrica no sul do Brasil. Postes em diferentes estados de deterioração foram retirados da rede para os ensaios de flexão estática e medidas de densidade. Os testes mecânicos indicaram que os valores médios da resistência a flexão e da rigidez decrescem com o estado de deterioração do eucalipto. Entretanto, verificou-se uma grande variação nessas medidas com diferentes classes de deterioração apresentando resistência mecânica residual similar. Esse comportamento pode ser explicado pela influência da densidade da madeira nas correlações entre propriedades mecânicas e o estado de deterioração dos postes. Madeiras menos densas podem apresentar baixa resistência mecânica residual mesmo sem apresentar sinais de deterioração. O estudo da caracterização da madeira de eucalipto indicou que o estado de deterioração avaliado pela inspeção em campo, por si só não é um bom indicativo para prever a durabilidade das estruturas de madeiras, fazendo-se necessária uma análise preliminar da densidade da mesma.
\end{abstract}

Palavras-chave: Eucalipto, Deterioração e Densidade.

\section{RELATIONSHIP BETWEEN MECHANICAL PROPERTIES AND DENSITY OF} THE EUCALYPTUS WOOD POLES WITH THEIR DETERIORATION STATURS

\begin{abstract}
This paper presents the correlations obtained among mechanical properties, density and deterioration status of new eucalyptus poles and those removed from service. The deterioration status was determined in the field in sections of poles of the distribution of electric energy in southern Brazil. Poles in various status of deterioration were removed from the network for static bending tests and density measurements. The mechanical tests indicated that the average values of bending strength and stiffness decline with the deteriorating state of eucalyptus. However, there was a large variation in these measures with different classes of deterioration presenting similar residual mechanical strength. This behavior can be explained by the influence of wood density on the correlations between mechanical properties and the state of deterioration of the poles. Less dense woods can have residual mechanical strength low even without present signs of deterioration. The study of the characterization of eucalyptus wood indicated that the state of deterioration measured by field inspection alone is not a good indicator for predicting the durability of wood structures, making necessary a preliminary analysis of the density.
\end{abstract}

Keywords: Eucalyptus, Deterioration and density..

\footnotetext{
${ }^{1}$ Recebido em 08.12.2011 aceito para publicação em 04.06.2012

${ }^{2}$ Pós-Graduação em Engenharia e Tecnologia de Materiais, Pontifícia Universidade Católica do Rio Grande do Sul, PUCRS, Brasil. E-mail: <rafael.abruzzi@acad.pucrs.br>.

${ }^{3}$ Pontifícia Universidade Católica do Rio Grande do Sul, Faculdade de Química, Departamento de Química Aplicada. E-mail: $<$ mpires@pucrs.br>.

${ }^{4}$ Pontifícia Universidade Católica do Rio Grande do Sul, Faculdade de Engenharia, Departamento de Engenharia Mecânica e Mecatrônica. E-mail: <berenice@ pucrs.br>.

${ }^{5}$ Pontifícia Universidade Católica do Rio Grande do Sul, Escola Politécnica, Departamento de Engenharia Civil.. E-mail: <silvia.kalil@pucrs.br>.
} 


\section{INTRODUÇÃO}

Entre os diversos usos da madeira, cabe destacar a fabricação de postes que servem de suporte às redes de energia e telefonia. No Brasil, principalmente nos Estados da Região Sul, o uso de postes de eucalipto representa cerca de $90 \%$ do total dos postes em serviço na rede de distribuição de energia elétrica (VIDOR et al., 2009). Essas estruturas são fabricadas a partir de florestas plantadas de diversas espécies de eucalipto e de outros povoamentos cultivados com grande diversidade de espécies também para outros fins. Postes de eucalipto, em geral, possuem excelente resistência mecânica, baixa densidade e custo reduzido de fabricação quando comparados com postes de aço e de concreto.

A utilização eficiente de estruturas de qualquer material depende, principalmente, da compreensão do seu comportamento mecânico. Assim, a determinação das propriedades mecânicas na madeira, como elasticidade e resistência, deve ser feita de forma criteriosa, pois se trata de um material heterogêneo (KRETSCHMANN, 2010).

Devido à baixa resistência à biodeterioração, a madeira deve ser preservada quimicamente para garantir um tempo mínimo de vida útil de 15 anos estabelecido pela NBR 8456 (1984) (LEPAGE, 1986; MORRELL, 1996). Entretanto, segundo Vidor et al. (2009), mesmo tratadas, determinadas espécies de eucalipto apresentam diminuição acelerada no estado de conservação associada à negligência, tanto no processo de fabricação quanto na seleção das espécies de eucalipto.

Tratamentos curativos, bem como a inspeção e manutenção periódicas dos postes em serviço, têm sido propostos para minimizar problemas com a deterioração da madeira. Vidor (2011) relatou que procedimentos de inspeção tradicionais, utilizados até agora, não têm sido eficientes em avaliar as condições de resistência residual dos postes em serviço.

A norma NBR 6231 (ABNT, 1980) estabelece alguns parâmetros para qualificar postes de madeira que irão ser colocados em serviço. Cabe destacar os testes destrutivos de flexão, por amostragem, através do método denominado viga engastada em balanço, que devem ser realizados para garantir a resistência mecânica mínima para o poste. Porém, poucos autores relataram sobre a resistência residual da madeira após anos de serviço na rede. Os parâmetros característicos considerados para o dimensionamento de postes novos de eucalipto, conforme a NBR 8456 (1984), são: Limite de Resistência à Flexão (MORf) de 85 MPa e Módulo de Elasticidade à Flexão (MOEf) de 13.000 MPa.

A NBR 8456 (1984) aborda informações das propriedades físicas e mecânicas da madeira, em que parametriza o MORf e o MOEf dentro das espécies de eucalipto, considerando suas densidades aparentes. Já conforme a proposta de revisão dessa mesma norma, o MORf e o MOEf devem ser parametrizados, considerando-se nove classes de densidade básica que variam desde 400 a mais que $800 \mathrm{~kg} / \mathrm{m}^{3}$, e a resistência varia de 44 a $94 \mathrm{MPa}$, respectivamente, entre a primeira e a última classe de densidade.

Autores como Kretschmann (2010) e Evans et al. (2000) relacionaram a resistência mecânica com as características naturais da madeira. Variações no tamanho dos poros e na espessura das paredes da célula fazem que algumas espécies de madeira tenham mais substâncias por unidade de volume do que outras espécies e, portanto, maior densidade. A presença de extrativos e o teor de umidade também podem influenciar nas propriedades mecânicas da madeira.

Segundo Shimoyama (1990), a densidade está relacionada a muitas propriedades e características tecnológicas fundamentais para a produção e utilização da madeira, sendo um dos parâmetros mais importantes entre as diversas propriedades físicas da madeira, pois afeta grande parte das suas demais propriedades.

Portanto, o objetivo deste trabalho foi verificar a correlação das propriedades mecânicas residuais (MORf e MOEf) com a densidade e com o estado de deterioração obtido através de parâmetros classificatórios usuais, em postes com diferentes tempos em serviço.

\section{MATERIAL E MÉTODOS}

\subsection{Inspeção de Postes de Eucalipto}

A metodologia para a inspeção apresentada neste trabalho foi validada por Vidor (2011), baseada em normas de inspeção nacionais e internacionais, como OSHA (2003) e ASTM D390 (ASTM, 1996). Primeiro, é realizada uma inspeção visual da estrutura, à procura de rachaduras ou falhas, além da escavação na sua base. Após, com um martelo, é realizada a inspeção por percussão, e a resposta sonora identifica regiões com apodrecimento interno. Em seguida, é realizada uma escavação no solo, ao redor do poste, para a verificação externa da estrutura 
em sua região de engastamento. Na linha do solo, é realizada uma raspagem da superfície, na região externa, a fim de determinar o volume de madeira apodrecida. Um furo é realizado na região, de modo a prosseguir com a inspeção interna. Com uma escala graduada, é quantificado o volume de madeira interna ainda em boas condições.

A partir dos dados obtidos no processo de inspeção, foram adotados critérios quantitativos de decaimento, propondo uma classificação para o poste inspecionado baseada numa escala numérica (de 1 a 4) e de cores. Ações foram propostas para cada situação: 1. (verde) poste considerado em bom estado, nenhuma ação corretiva sugerida; 2. (amarelo) decaimento parcial, mas o poste pode continuar em serviço e um retratamento interno/externo é recomendado; 3. (laranja) poste rejeitado com avançado decaimento, um reforço/retratamento deve ser feito; 4. (vermelho): perigo, poste rejeitado, não podendo permanecer em serviço; necessita de substituição imediata.

\subsection{Amostragem de Postes Para Testes de Resistência Mecânica e Densidade}

A seleção dos postes coletados para os testes de flexão e determinação da densidade foi baseada na classificação realizada durante as inspeções. Entre os postes inspecionados, cerca de 800 , com tempo de serviço variando de 3-33 anos, foram retirados da rede elétrica para execução de testes destrutivos de verificação da resistência mecânica residual através de testes de flexão estática, porém somente 336 ensaios puderam ser validados devido à má conservação dos demais. A seleção dos postes coletados para os testes de resistência mecânica foi baseada na sua classificação (estados de deterioração), distribuídos conforme apresentado na Tabela 1.

Tabela 1 - Quantidade de postes submetidos aos testes de flexão. Table 1 -Number of poles submitted to bending tests.

\begin{tabular}{|c|c|c|c|c|c|c|}
\hline \multicolumn{4}{|c|}{ Classe } & \multirow{2}{*}{$\begin{array}{c}\text { Sem } \\
\text { Classe* }\end{array}$} & \multirow{2}{*}{$\begin{array}{c}\text { Sem } \\
\text { Tratamento }\end{array}$} & \multirow[t]{2}{*}{ Total } \\
\hline 1 & 2 & 3 & 4 & & & \\
\hline & & & & $\mathrm{CCA}$ & & \\
\hline \multirow[t]{2}{*}{61} & 52 & 30 & 33 & 24 & & 200 \\
\hline & & & & Creosoto & & \\
\hline \multirow[t]{2}{*}{32} & 26 & 21 & 37 & 14 & & 130 \\
\hline & & & & Total & & \\
\hline 93 & 78 & 51 & 70 & 38 & 6 & 336 \\
\hline
\end{tabular}

*Postes dos quais não se têm a classe de deterioração.
Postes tratados com arseniato de cobre cromatado (CCA) (tratamento hidrossolúvel) e creosoto (tratamento oleossolúvel não mais utilizado em postes desde 1998) foram considerados neste estudo.

Para determinação da densidade aparente foi selecionada uma amostragem de 69 postes, sendo 19 (28\%) classe $1,18(26 \%)$ classe $2,12(17 \%)$ classe 3 e $12(17 \%)$ pertenciam à classe 4 , visando contemplar todas as classes de deterioração. Foram também testados oito (12\%) postes novos das espécies E. grandis, $E$. saligna, E. tereticornis e C. citriodora, visando a uma comparação entre as propriedades mecânicas.

De acordo com Oliveira et al. (2005), a variação longitudinal da densidade da madeira de eucalipto não é significativa para muitas espécies, mas não se apresenta de forma uniforme. Em razão disso, as amostras para os testes de densidade foram coletadas de uma mesma região do poste, logo acima da linha de afloramento, interseção da superfície lateral do poste com o plano do solo.

\subsection{Testes de Resistência Mecânica}

Os testes destrutivos de flexão foram realizados em uma área de testes especialmente implementada para esse fim, instalada no campus da PUCRS. Neste estudo, os testes foram executados em postes retirados das redes em diferentes estados de deterioração e densidade. Seguiram-se a metodologia e os parâmetros descritos nas NBR6231 (Postes de Madeira - Resistência à Flexão) e NBR 8456 (Postes de Eucalipto Preservados para Redes de Distribuição de Energia Elétrica), que se referem a postes novos.

O teste de flexão foi iniciado com o poste perfeitamente engastado no equipamento e fixo de forma a não executar qualquer movimento nesse ponto no momento da flexão. A carga é aplicada continuamente no topo do poste, até que ocorra sua ruptura, de tal forma que a sua velocidade de deformação seja constante. O limite de resistência à flexão da madeira (MORf) na seção de engastamento e o módulo de elasticidade à flexão (MOEf) foram determinados conforme orientação da NBR 6231 (ABNT, 1980).

\subsection{Determinação da Densidade Aparente da Madeira}

Seções de aproximadamente $0,50 \mathrm{~m}$ de comprimento, cortadas logo acima da provável região de intersecção do poste com o solo, foram utilizadas para a confecção

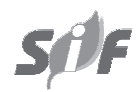

Revista Árvore, Viçosa-MG, v.36, n.6, p.1173-1181, 2012 
dos corpos de prova de 150 x 50 x 50 mm, para a determinação da densidade aparente pelo método estereométrico segundo as orientações da NBR 7190 (ABNT, 1997).

\subsection{Análise Estatística e Cálculos}

O grau de associação entre as variáveis estudadas foi medido através do coeficiente de correlação de Pearson, que é o modelo pelo qual se avaliam os dados para comprovar se uma das variáveis é explicada pela outra através de uma reta para dados de uma amostra. Com isso, podem-se verificar correlações entre estado de deterioração, MORf, MOEf e densidade. Adotando-se um $p$-valor de 0,05, ou seja, um nível de significância de $5 \%$.

\section{RESULTADOS}

A relação entre as propriedades mecânicas residuais (MORf e MOEf), obtidas nos ensaios de flexão estática com os diferentes estados de deterioração da madeira de 336 postes que foram inspecionados em campo e logo depois de retirados de serviço, é apresentada na Figura 1a. Considerando somente a média dos valores, verificou-se redução do MORf, com o aumento da classe, ou seja, com o decréscimo da qualidade dos postes testados. O mesmo ocorreu com o MOEf em razão da classe de postes testados, conforme mostrado na Figura $1 \mathrm{~b}$.

A Figura 1c apresenta o comportamento do valor médio do MORf para postes nas quatro diferentes classes de deterioração e para os dois tipos de tratamento utilizados (CCA e creosoto). Fica evidente a maior resistência nos postes tratados com creosoto, em comparação com os tratados com CCA. Verificou-se, também, decréscimo do MORf conforme ocorria o aumento da classe de deterioração, principalmente para o creosoto. $\mathrm{O}$ mesmo aconteceu para o MOEf nos postes trados com creosoto e CCA, conforme mostrado na Figura 1d.

Com as correlações de Pearson entre as propriedades mecânicas e os diferentes estados de deterioração da madeira de eucalipto, verificou-se forte correlação entre as duas propriedades mecânicas estudadas $(0,72)$ e uma fraca correlação entre as propriedades mecânicas e as diferentes classes de deterioração (-0,28 para MORf e $-0,22$ para MOEf).

Como os dados de inspeção não apresentaram correlação satisfatória com as propriedades mecânicas, 69 postes foram selecionados para investigar, mediante os ensaios de densidade em laboratório, a influência desta nas propriedades mecânicas da madeira.

O comportamento dos 61 postes retirados de serviço separados por classe, ou seja, postes com semelhantes estados de deterioração, diante do teste de flexão estática, é apresentado na Figura 2. Nesta, verifica-se correlação linear positiva entre o MORf e a densidade da madeira, o que fica evidenciado com a correlação de Pearson apresentada na Tabela 2, com valores em torno de 0,70 para os postes retirados de serviço, comprovados pelo $p$-valor, sendo todos inferiores a 0,05 , ou seja, menores que o nível de significância de $5 \%$.

Analisando a Figura 2e, que apresenta o comportamento dos oito postes novos diante do teste de flexão, verifica-se a mesma tendência de correlação linear positiva entre o MORf e a densidade da madeira, o que fica evidenciado com a correlação de Pearson apresentada na Tabela 2 , com valor de 0,94 para os postes novos, comprovados pelo $p$-valor, sendo inferior a 0,05 , ou seja, menor que o nível de significância de $5 \%$.

Verificou-se, comportamento semelhante das propriedades mecânicas também para o MOEf com relação à densidade da madeira nos postes retirados de serviço. Esse fato ficou evidenciado com a correlação de Pearson para MOEf apresentada na Tabela 2, com valores em torno de 0,60 dentro de cada classe de deterioração, comprovados pelo $p$-valor, sendo todos inferiores a 0,05 , ou seja, menores que o nível de significância de 5\%. A mesma tendência foi observada nos postes novos, o que ficou evidente com a correlação de Pearson apresentada na Tabela 2, com valor de 0,74 , comprovados pelo $p$-valor, sendo inferior a 0,05 , ou seja, menor que o nível de significância de $5 \%$. Verifica-se também, com acorrelaçãodePearson, conforme observado na Tabela 2, que, mesmo sem estarem separados por classes de deterioração, ficou clara a correlação linear positiva do MORf e do MOEf, com a densidade da madeira com valores de correlação de 0,67 e 0,66 , respectivamente, e também com $p$-valor extremamente baixo.

Também se observa, nessa tabela, correlação entre as propriedades mecânicas em três das classes de deterioração, mas de forma menos intensa. Novamente uma correlação fraca (-0,36 para MORf e -0,32 para MOEf) foi verificada entre as classes de deterioração e as propriedades mecânicas analisadas, o que ressalta a importância da análise da densidade das estruturas da madeira. 


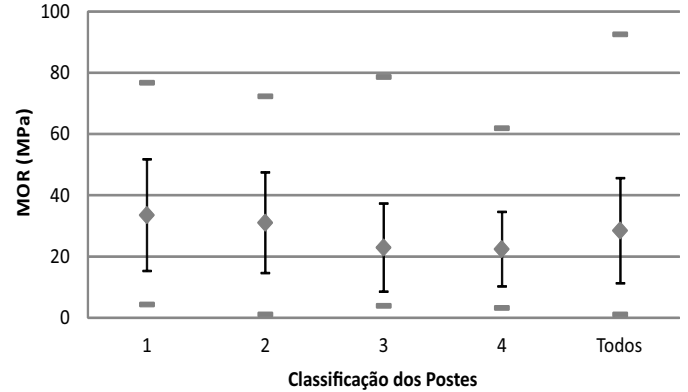

- Média -Mín. -Máx.

a)

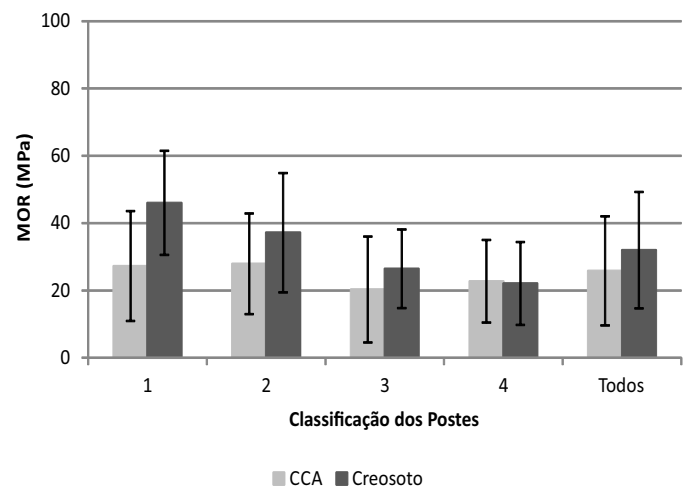

c)

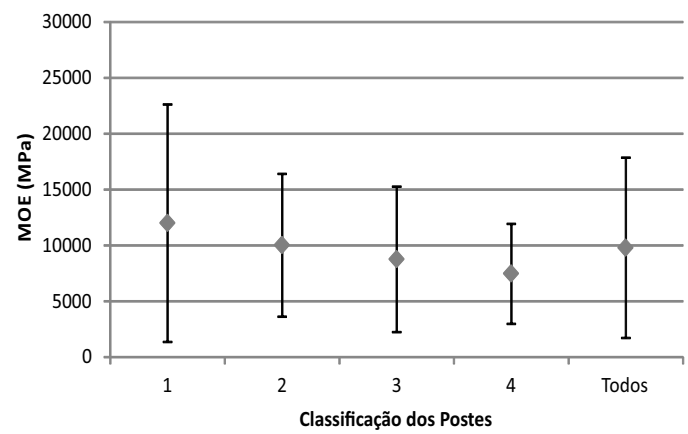

b)

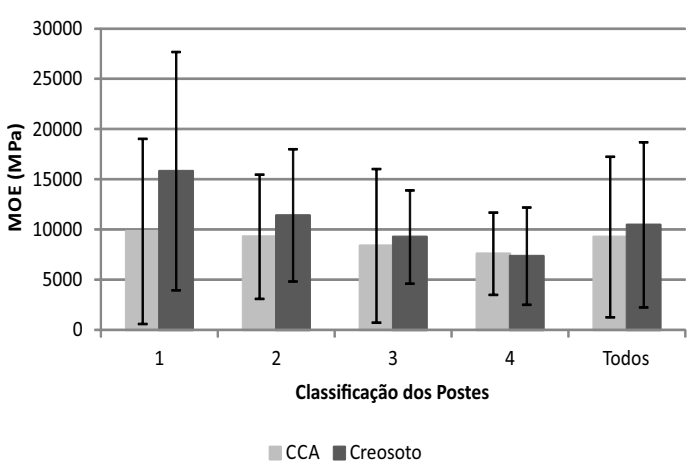

d)

Figura 1 - Variação média do Limite de Resistência à Flexão (MORf) e módulo de elasticidade à flexão (MOEf) onde, a) e b) Postes retirados de serviço conforme seu estado de deterioração, c) e d) Postes retirados de serviço conforme seu tratamento e estado de deterioração.

Figure 1 - Average variation of Bending Strength Limit (MORf) and bending modulus of elasticity (MOEf), where a) and b) Poles removed from service by their status of deterioration, $c$ ) and d) Poles removed from service as their treatment and status of deterioration.

\section{DISCUSSÃO}

Resultados incoerentes foram verificados entre o estado de deterioração estimado nas inspeções e a resistência residual obtida nos testes destrutivos de flexão de postes retirados de serviço. O mesmo foi observado por Vidor e colaboradores (2011). Variações significativas com elevados desvios-padrão nas medidas de MORf e, principalmente, de MOEf na Figura 1 ab evidenciam que postes com deterioração mais elevada apresentam maior resistência residual que alguns postes de melhor estado de deterioração. Dentro de uma mesma classe de deterioração, também foram verificadas variações significativas com elevados desvios-padrão para MORf e MOEf, indicando

não esta nas referências elevada variação de resistência residual do poste em serviço. Tal comportamento sugere grande variabilidade de resistências mecânicas dos postes na rede estudada, que pode estar relacionada ao uso de diferentes espécies de eucaliptos de densidades muito distintas.

A fraca correlação entre as propriedades mecânicas e as classes de deterioração da madeira de eucalipto, obtidas com a inspeção em campo e apresentadas na Tabela 2, corroboram esses dados.

Durante as inspeções foram encontrados postes antigos e bem conservados, com valores de MORf e MOEf mais elevados, a maioria tratado com Creosoto, conforme

Revista Árvore, Viçosa-MG, v.36, n.6, p.1173-1181, 2012 
Postes Classe 1

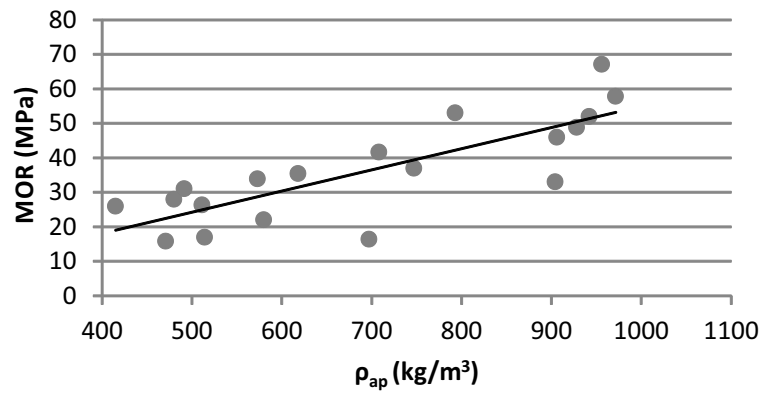

a)

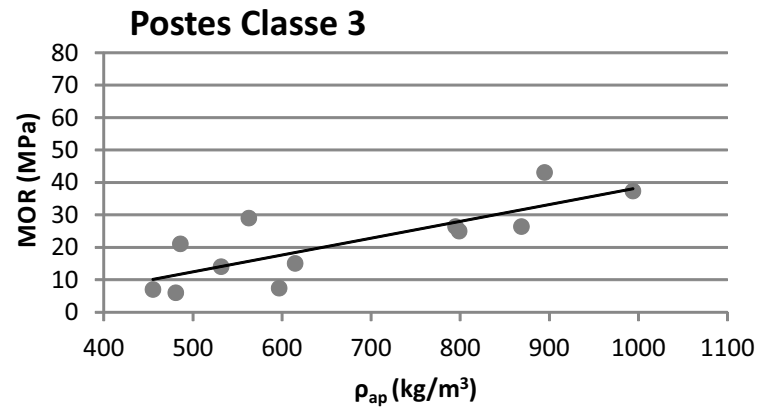

c)

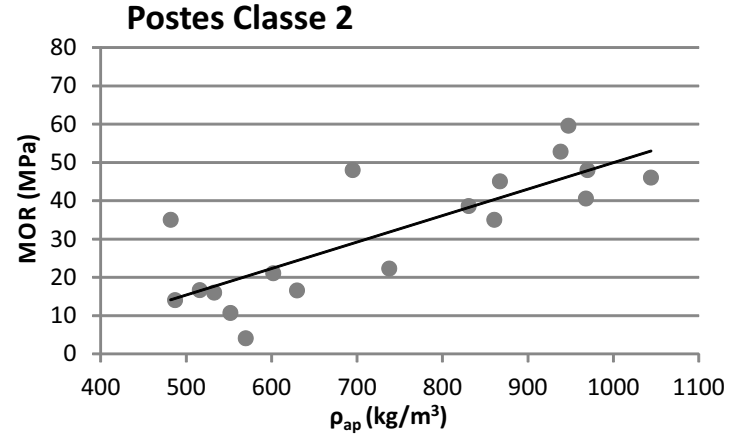

b)

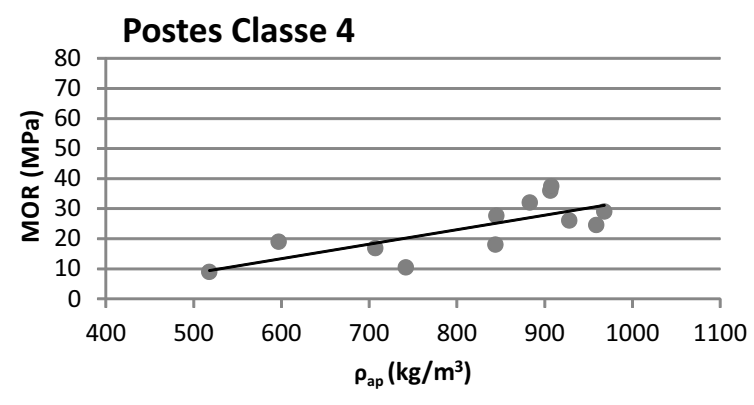

d)

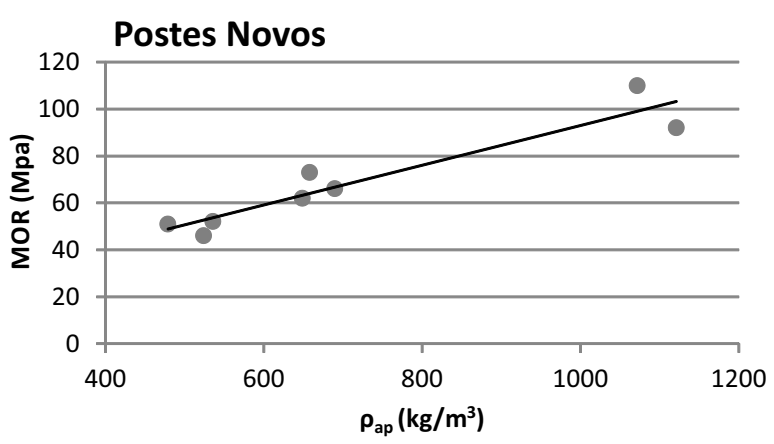

e)

Figura 2 - Variação individual do Limite de Resistência à Flexão (MORf) em relação a densidade para postes retirados de serviço onde, a) Postes da classe 1, b) Postes da classe 2, c) Postes da classe 3, d) Postes da classe 4 e e) Postes novos.

Figure 2 -Individual variation of the Bending Strength Limit (MORf) in relation to the density poles removed from service where a) Class 1 poles, b) Class 2 Poles, c) Class 3 poles, d) Class 4 poles and e) New poles.

apresentado na Figura $1 \mathrm{~cd}$. Porém, neste caso uma análise mais criteriosa, deve levar em consideração que quando o Creosoto estava sendo utilizado como preservante para postes, a madeira utilizada na época era obtida de espécies de alta densidade, em relação a outras espécies de eucalipto. Este fator pode estar contribuindo para que postes tratados com Creosoto ainda estejam na rede e apresentem uma boa resistência residual média.

Revista Árvore, Viçosa-MG, v.36, n.6, p.1173-1181, 2012 
Tabela 2 - Correlações de Pearson entre as propriedades mecânicas e a densidade da madeira de 69 postes. Table 2 - Pearson correlations between the mechanical properties and wood density of 69 poles.

\begin{tabular}{|c|c|c|c|c|c|c|}
\hline & \multicolumn{3}{|c|}{ Matriz de Correlação: Pearson } & \multicolumn{3}{|c|}{ Matriz de $p$-valores } \\
\hline & MOR & MOE & $\rho_{\text {ap }}$ & MOR & MOE & $\rho_{\text {ap }}$ \\
\hline \multicolumn{7}{|c|}{ Postes Classe 1} \\
\hline MOR & 1 & & & 1 & & \\
\hline MOE & 0,75 & 1 & & 0,00029 & 1 & \\
\hline$\rho_{\text {ap }}$ & 0,79 & 0,58 & $\begin{array}{c}1 \\
\text { Postes Classe } 2\end{array}$ & 0,00011 & 0,01 & 1 \\
\hline MOR & 1 & & & 1 & & \\
\hline MOE & 0,46 & 1 & & $* 0,06$ & 1 & \\
\hline$\rho_{\mathrm{ap}}$ & 0,77 & 0,69 & $\begin{array}{c}1 \\
\text { Postes Classe } 3\end{array}$ & 0,00042 & 0,0033 & 1 \\
\hline MOR & 1 & & & 1 & & \\
\hline MOE & 0,89 & 1 & & 0,00027 & 1 & \\
\hline$\rho_{\mathrm{ap}}$ & 0,77 & 0,68 & $\begin{array}{c}1 \\
\text { Postes Classe } 4\end{array}$ & 0,005 & 0,021 & 1 \\
\hline MOR & 1 & & & 1 & & \\
\hline MOE & 0,69 & 1 & & 0,014 & 1 & \\
\hline$\rho_{\text {ap }}$ & 0,75 & 0,64 & $\begin{array}{c}1 \\
\text { Postes Novos }\end{array}$ & 0,0047 & 0,025 & 1 \\
\hline MOR & 1 & & & 1 & & \\
\hline MOE & 0,52 & 1 & & $* 0,19$ & 1 & \\
\hline$\rho_{\mathrm{ap}}$ & 0,94 & 0,74 & $\begin{array}{c}1 \\
\text { Todos os Postes }\end{array}$ & 0,00045 & 0,037 & 1 \\
\hline MOR & 1 & & & 1 & & \\
\hline MOE & 0,7 & 1 & & $2,84 \mathrm{E}-06$ & 1 & \\
\hline$\rho_{\text {ap }}$ & 0,67 & 0,66 & 1 & $2,92 \mathrm{E}-09$ & $1,86 \mathrm{E}-05$ & 1 \\
\hline Classe & $-0,36$ & $-0,32$ & 0,16 & 0,04 & 0,02 & 0,23 \\
\hline
\end{tabular}

*Valores não significativos.

De acordo com os resultados deste trabalho, as propriedades mecânicas são notavelmente influenciadas pela densidade da madeira de eucalipto. Comportamento semelhante foi evidenciado por Lobão et al. (2004) e Armstrong (1984), que também apontaram alto nível de confiança entre a densidade e as propriedades mecânicas. Propriedades mecânicas em razão da densidade da madeira também foram avaliadas por Zhang (1994), em que análises de espécies do gênero Eucalyptus indicaram relação linear entre as propriedades mecânicas, como MOR e MOE, e a densidade.

Apesar de a correlação do MOEf ser mais fraca que a correlação do MORf com a densidade, fica evidenciado que ambos são influenciados por essa correlação, sendo o mesmo observado por Zhang (1997).

A relação entre as propriedades mecânicas e a densidade separada por estados de deterioração, apresentadas na Figura 2a-d, evidencia risco maior para os postes de menor densidade, uma vez que possuem menor resistência residual. As variações significativas nas propriedades mecânicas obtidas para os postes novos (Figura 2e) mostram que a maioria já apresenta um limite de resistência à flexão menor do que $85 \mathrm{MPa}$, o mínimo exigido pela NBR 8456 (1984), sendo estes os de menor densidade.

Observou-se a importância da determinação da densidade da madeira para complementar o método de inspeção, visando a uma estimativa da resistência residual dos postes, uma vez que somente com a inspeção não se consegue avaliar, de forma efetiva, a resistência residual desse poste.

Diante dos resultados, pode-se sugerir que o processo de inspeção deve ser complementado com a coleta de pequenas amostras de madeira para realização dos testes de densidade em laboratório. A coleta pode ser realizada com o auxílio de uma broca oca adaptada a um perfurador 
de madeira a bateria. Esse equipamento permite a retirada de cilindros (baguetas) com diâmetros e comprimentos variando conforme o tipo e penetração da broca, e a coleta pode ser realizada no mesmo orifício da inspeção, seguida de retratamento no local perfurado, apresentado por Vidor (2011).

\section{CONCLUSÕES}

Os resultados da caracterização da madeira indicaram que os valores médios da resistência à flexão e da rigidez decrescem com o estado de deterioração do eucalipto, mas esse decaimento está fortemente relacionado com a densidade, ou seja, com a espécie de eucalipto empregada, mesmo para postes com longo tempo em serviço, em que os postes mais densos apresentam maior resistência mecânica residual.

A relação das propriedades mecânicas com a densidade sugere que o estado de deterioração por si só não é um bom indicativo para prever a durabilidade da estrutura de madeira, fazendo-se necessária uma análise preliminar da sua densidade.

Quanto ao uso da madeira, viu-se a necessidade de avaliação preliminar dos valores de densidade, a fim de que o uso dessa madeira seja compatível com as resistências necessárias para as utilizações correspondentes. As espécies de maior densidade são mais recomendadas quando se desejam maior durabilidade e resistência mecânica da estrutura.

\section{AGRADECIMENTOS}

AAES Sul pelo financiamento, a CAPES e a PUCRS.

\section{REFERÊNCIAS}

\section{ASSOCIAÇÃO BRASILEIRA DE NORMAS} TÉCNICAS- ABNT. NBR 6231: Postes de madeira - resistência à flexão. Rio de Janeiro: 1980. 4p.

\section{ASSOCIAÇÃO BRASILEIRA DE NORMAS} TÉCNICAS - ABNT. NBR 8456: Postes de eucalipto preservado para redes de distribuição de energia elétrica. Rio de Janeiro: 1984. 31p.

\section{ASSOCIAÇÃO BRASILEIRA DE NORMAS} TÉCNICAS - ABNT. NBR 7190: Projeto de estruturas de madeira. Rio de Janeiro: 1997. 107p.
ARMSTRONG, J. P.; SKAAR, C.; DEZEEUW, C. The effect of specific-gravity on several mechanical-properties of some world woods. Wood Science and Technology, v. 18, n. 2, p. 137-146, 1984.

\section{AMERICAN SOCIETY FOR TESTING AND MATERIALS -ASTMD 390- 96,Designation.Philadelphia: 1996.}

EVANS, J. W.; SENFT, J. F.; GREEN, D. W. Juvenile wood effect in red alder: Analysis of physical and mechanical data to delineate juvenile and mature wood zones. Forest Products Journal, v. 50, n. 7-8, p. 75-87, 2000.

KRETSCHMANN, D. E. Mechanical properties of wood In:BERGMAN, R., et al.Wood handbook. Washington: USDA, 2010. p.100-145.

LEPAGE, E. S. Manual de preservação de madeiras. São Paulo:Instituto de Pesquisas Tecnológicas, 1986. 2v.

LOBÃO, M. S. et al. Caracterização das propriedades físico-mecânicas da madeira de eucalipto com diferentes densidades. Revista da Árvore, v.28, n.6, p.889-894, 2004.

MORRELL, J. J. Wood pole maintenance manual.Corvallis: Forest Research Laboratory, Oregon State University, 1996.

OSHA.Methodsofinspectingandtestingwood poles. 1910.269 App D, 2003.

OLIVEIRA, J. T. S.; HELLMEISTER, J. C.; TOMAZELLO FILHO, M. Variação do teor de umidade e da densidade básica na madeira de sete espécies de eucalipto. Revista da Árvore, v.29, n.1, p.115-127, 2005.

SHIMOYAMA, V. R. S. Variações da densidade básica e características anatômicas e químicas da madeira em Eucalyptus spp. 1990. 93f. Dissertação (Mestrado em Ciências Florestais) Escola Superior de Agricultura Luiz de Queiroz, Piracicaba, 1990.

VIDOR, F. L. R. et al.Inspection of wooden poles in electrical power distribution networks in southern Brazil. IEEE Transactionson Power Delivery, v.25, n.4, p.479-484, 2009. 
VIDOR, F. L. R. Avaliação da vida útil de postes de madeira de eucalipto em serviço em redes de distribuição de energia elétrica. 2011. 130f. Tese (Doutorado em Engenharia e Tecnologia de Materiais) - Faculdade de Engenharia, Física e Química, Porto Alegre, 2011.
ZHANG, S. Y. Mechanical-properties in relation to specific-gravity in 342 chinese woods. Wood and Fiber Science, v.26, n.4, p.512-526, 1994.

ZHANG, S. Y. Wood specific gravity mechanical property relationship at species level. Wood Science and Technology, v.31, n.3, p.181-191, 1997. 
